\title{
Effects of FSTL1 on cell proliferation in breast cancer cell line MDA-MB-231 and its brain metastatic variant MDA-MB-231-BR
}

\author{
JIAQIANG AN ${ }^{1 *}$, LULU WANG $^{1-3^{*}}$, YUANLI ZHAO ${ }^{4}$, QIANG HAO $^{4}$, YING ZHANG ${ }^{1}$, JINGYI ZHANG $^{1}$, \\ CHUN YANG ${ }^{1}$, LI LIU ${ }^{1}$, WENJUAN WANG ${ }^{1}$, DONGLIANG FANG ${ }^{1}$, TAO LU $^{1}$ and YAN GAO ${ }^{1-3}$ \\ ${ }^{1}$ Department of Human Anatomy, Capital Medical University; ${ }^{2}$ Beijing Key Laboratory of Cancer Invasion \\ and Metastasis Research; ${ }^{3}$ Cancer Institute of Capital Medical University; ${ }^{4}$ Department of Neurosurgery, \\ Beijing Tian Tan Hospital, Capital Medical University, Beijing 100069, P.R. China
}

Received February 24, 2017; Accepted September 4, 2017

DOI: $10.3892 /$ or.2017.6004

\begin{abstract}
In the past decades, altered Follistatin-like 1 (FSTL1) expression has been documented in a variety of cancers, while its functional roles are poorly understood. Particularly in breast cancer, the expression of FSTL1 and its signaling pathway remain to be determined. In the present study, an elevated FSTL1 expression and a supressed cell proliferation were detected in a specific brain metastatic cell line MDA-MB-231-BR (231-BR), compared with its parental cell line MDA-MB-231. However, this protein was hardly detected in the other three breast cancer cell lines. Next, lentiviral vectors encoding FSTL1 or FSTL1 specific shRNAs were used to overexpress or knock down FSTL1 in MDA-MB-231 or 231-BR, respectively (MDA-MB-231 ${ }^{\mathrm{FSTL} 1}$ or 231-BR ${ }^{\mathrm{sh} F S T L 1}$ ). Results showed that overexpression of FSTL1 inhibited MDA-MB-231 cell proliferation, while knockdown of FSTL1 in 231-BR cells promotes cell proliferation, compared with their corresponding control groups. These results were further confirmed in nude mouse xenografts. The tumor volume in 231-BR cell-bearing mice was significantly smaller than that of MDA-MB-231 group, and reduction of tumor volume was detected in MDA-MB-231 ${ }^{\mathrm{FSTL}}$ cell-bearing mice compared with the control group. Previous studies revealed that TGF- $\beta$ Smad2/3 signaling pathway was activated in 231-BR and
\end{abstract}

Correspondence to: Dr Yan Gao, Department of Human Anatomy, Capital Medical University, Beijing 100069, P.R. China

E-mail:gy1003@ccmu.edu.cn

${ }^{*}$ Contributed equally

Abbreviations: 231-BR, MDA-MB-231-BR; BCBM, breast cancer brain metastases; BMP, bone morphogenetic protein; FSTL1, Follistatin-like 1; FRP, follistatin related protein; IHC, immunohistochemistry; RTCA, real-time cellular analysis; shRNA, short-hairpin RNA; TGF- $\beta$, transforming growth factor- $\beta$; TSC-36, TGF- $\beta 1$ stimulated clone 36

Key words: Follistatin-like 1, breast cancer, brain metastasis, proliferation, TGF- $\beta$
MDA-MB-231 ${ }^{\text {FSTL1 }}$ cells, which may contribute to the inhibited cell proliferation. In addition, Smad 3 knockdown could restore the inhibition of cell proliferation induced by FSTL1 overexpression in MDA-MB-231 ${ }^{\mathrm{FSTL} 1}$ cells, indicating that the anti-proliferative effect of FSTL1 overexpression may be associated with Smad3 involved TGF- $\beta$ signaling pathway regulation. This study identified FSTL1 as an inhibitor of cell proliferation in MDA-MB-231 and 231-BR cell lines, which may provide new insights into the development and management of breast cancer.

\section{Introduction}

Breast cancer is the most prevalent cancer and the second most frequent cause of death in women (1). Approximately 10-30\% of patients with breast cancer are diagnosed with brain metastasis, which represents a particularly devastating consequence of breast cancer due to its high mortality (1-3). Although systemic therapy for breast cancer patients has been improved and overall survival has been prolonged, the incidence of brain metastasis is increasing. No effective treatment for breast cancer brain metastasis (BCBM) is available at present, and the overall survival is on the order of months (mean 17 and median 15 months) (4). Therefore, there is an urgent need to better understand the molecular basis of BCBM and explore new therapeutic agents $(3,5-8)$.

$\mathrm{BCBM}$ is a complex process that involves a series of well-defined steps. To better understand BCBM, 'brain-seeking' clonal sublines have been generated $(4,9,10)$. Among them, MDA-MB-231-BR (231-BR), the brain-seeking clones of MDA-MB-231, was derived by multiple selection rounds of brain metastatic lesions after intracardiac injection of MDA-MB-231 cells (9). Different from its parental cell line MDA-MB-231 which is highly metastatic but has no organ specificity, 231-BR metastasizes to the brain with $100 \%$ accuracy, making it a widely used preclinical model for brain metastatic breast cancer (11-16). On account of their syngeneic nature, proteome of the brain-seeking cell line has been compared with its parental cell lines, with the purpose of finding differentially expressed proteins that may play roles in the establishment or progression of BCBM (11). A few genes or proteins have been discovered, while the possibility that they 
can be served as potential biomarkers or targets for BCBM has not been confirmed yet $(4,11,17)$. Among them is the multifunctional cytokine transforming growth factor- $\beta 1$ (TGF- $\beta 1$ ), which has been extensively studied due to its various effects on carcinoma cell populations (18).

Initially known as TGF- $\beta 1$ stimulated clone 36 (TSC-36), Follistatin-like 1 (FSTL1) was first isolated from a mouse osteoblast cell line as a TGF- $\beta 1$-inducible gene (19). FSTL1 was reported as a regulator in embryonic organogenesis (20), a pro-inflammatory protein in rheumatoid arthritis (21), and a cardioprotective factor against ischemic injury (22). In the past decades, accumulating evidence has been obtained, suggesting a role of FSTL1 in cancer (23-28), while its functions are still poorly understood. Also, the signaling pathways involved in the expression of FSTL1 remain to be determined. Various, sometimes contradictory effects of FSTL1 have been demonstrated on cancer cell growth and survival (23-28). To date, the expression and the function of FSTL1 in breast cancer or BCBM has not been investigated. Only one study mentioned that the number of $\mathrm{ALCAM}^{+}$cells was correlated with the amount of tumor bone metastasis in a murine model, and $\mathrm{ALCAM}^{+}$cells correlatively increased in $\mathrm{FSTL1}^{+}$tumor tissues of patients with advanced breast cancer, which might suggest a possible role of FSTL1 in breast cancer, while further validation is required and signaling pathways involved need to be determined (24).

To determine the expression of FSTL1 in breast cancer, we detected its expression in four breast cancer cell lines, as well as a brain metastatic breast cancer cell line 231-BR. A higher level of FSTL1 was detected in 231-BR, meanwhile this protein was hardly detected in the other four breast cancer cell lines including the 231-BR's parental cell line (MDA-MB-231). These observations motivated us to investigate the possible role of FSTL1 in breast cancer progression.

In the present study, the expression of FSTL1 was determined in breast cancer cell lines. We reported for the first time that the increased expression of FSTL1 in 231-BR is accompanied with a decreased cell proliferation rate in MDA-MB-231. Our data identified FSTL1 as an inhibitor of metastatic breast cancer cell proliferation, which may provide new insights into the development and management of breast cancer and BCBM.

\section{Materials and methods}

Cell culture. The human MDA-MB-231-BR 'brain-seeking' breast cancer cell line (231-BR cells) was described previously (9). The 231-BR cell line transfected with enhanced green fluorescent protein (EGFP) was kindly provided by $\mathrm{Dr}$ Patricia S. Steeg (National Cancer Institute, Bethesda, MD, USA) (9). MDA-MB-231, and MCF7 cell lines were kindly provided by Dr Jun Wan (Hong Kong University of Science and Technology, Hong Kong, China). ZR-75-1, HCC38 cell lines were obtained from Dr Haili Qian (Chinese Academy of Medical Sciences, Beijing, China). MDA-MB-231, 231-BR and MCF7 cells were cultured in high-glucose Dulbecco's modified Eagle's medium (DMEM) (Gibco, Grand Island, NY, USA) containing 10\% fetal bovine serum (Gibco) and $1 \%$ penicillin-streptomycin (Gibco). ZR-75-1 and HCC38 cells were cultured in RPMI-1640 (Gibco) with $10 \%$ fetal bovine serum (Gibco) and 1\% penicillin-streptomycin (Gibco). All cells were cultured in $5 \% \mathrm{CO}_{2}$ at $37^{\circ} \mathrm{C}$.

Lentiviral construction. To stably overexpress FSTL1 in MDA-MB-231 cells, lentiviral vector (Ubi-MCS-3flag-RFPIRES-Puromycin) containing a FSTL1 coding sequence (NM_007085) was constructed (GeneChem, Shanghai, China). The empty vector was used as a control. To stably knock down FSTL1 in 231-BR cells, lentiviral vector (U6-MCS-Ubiquitin-Cherry-IRES-puromycin) containing the short-hairpin RNA (shRNA) specifically targeting FSTL1 or a negative control sequence was constructed (GeneChem). The following shRNA sequence targeting FSTL1 was used: TAAGGAGCAAATCCAAGAT. The following negative control sequence was used: TTCTCCGAACGTGTCACGT.

MTT. Cells were seeded into 96-well plate with the density of 2,000 cells per well at $37^{\circ} \mathrm{C}$ (day 0), allowed to adhere overnight (day 1) or continue in culture for another $24 \mathrm{~h}, 48 \mathrm{~h}$, $72 \mathrm{~h}$ or $96 \mathrm{~h}$. The cell culture medium was removed, and cells were incubated with $5 \mathrm{mg} / \mathrm{ml} \mathrm{3-(4,5-dimethylthiazol-2-yl)-}$ 2,5-diphenyltetrazolium bromide (MTT) (Sigma-Aldrich, St. Louis, MO, USA) for $3 \mathrm{~h}$, and then $100 \mu \mathrm{l}$ MTT solution (10\% Triton X-100, $0.1 \mathrm{~N} \mathrm{37.5 \%} \mathrm{HCl}, 90 \%$ isopropanol) was added per well. Absorbance was measured at $570 \mathrm{~nm}$. Data were normalized to day 1 .

Real-time cellular analysis (RTCA). To examine the proliferation of cells, E-plates and RTCA (RTCA-DP; ACEA Biosciences, USA) instrument was facilitated as described previously (29). Briefly, cells were seeded at a density of 2,000 cells per well in E-plates (3 wells repeated). Cell index was recorded every $15 \mathrm{~min}$ for $96 \mathrm{~h}$.

Colony forming assay. Colony forming assay was performed to examine the proliferation ability of cells. Cells were seeded into 6 -well plates at 400 cells per well ( 3 wells repeated), then cultured in $5 \% \mathrm{CO}_{2}$ incubator. After 7 days, the plates were washed with $0.01 \mathrm{M}$ PBS and stained by $0.1 \%$ crystal violet (Sigma-Aldrich) for $10 \mathrm{~min}$. The plates were washed with 0.01 M PBS 3 times and photographed using a microscope.

Western blotting. Cells were washed with ice-cold $0.01 \mathrm{M}$ PBS, lysed in the RIPA buffer with protease inhibitor and protein phosphatase inhibitor for $30 \mathrm{~min}$ on ice. Then cells were scraped and centrifuged with 12,000 rcf for $15 \mathrm{~min}$. Protein concentration was determined with BCA Protein assay kit (Thermo Scientific, Rockford, IL, USA). The proteins were separated by SDS-PAGE and transferred onto PVDF membrane. The primary antibodies used were Human FSTL1 Antibody (1:200, AF1694; R\&D Systems, Minneapolis, MN, USA), GAPDH (1:3,000, \#5174; Cell Signaling Technology Inc., Beverly, MA, USA), Smad2/3 (D7G7) $\mathrm{XP}^{\circledR}$ Rabbit mAb $(1: 1,000$, \#8685; Cell Signaling Technology Inc.), anti-phospho-Smad2/3 (pThr8) (1:1,000, SAB4504208; Sigma-Aldrich), anti-Smad1/5/8 antibody (1:200, sc-6031-R; Santa Cruz Biotechnology, Santa Cruz, CA, USA); anti-p-Smad1/5/8 (Ser 463/Ser 465) antibody (1:200, sc-12353; Santa Cruz Biotechnology); anti-Smad3 antibody (1:1,000, ab28379; Abcam, Cambridge, MA, USA); anti-Smad3 
(phospho S423+S425) antibody [EP823Y] (1:2,000, ab52903; Abcam); caspase-3 antibody (1:1,000, \#9662; Cell Signaling Technology Inc.); cleaved caspase-3 (Asp175) (5A1E) (1:1,000, \#9664; Cell Signaling Technology Inc.).

Real time-PCR. Total cellular RNA was extracted using Tri reagent (Sigma-Aldrich), and cDNA was generated using SuperRT cDNA kit (CWBio, China) as previously described (30). Specific primers for human FSTL1 (forward, 5'-CCAGACC ACGATGTGGAAAC-3'; reverse, 5'-GGCACAGATCTTGG ATTTGC-3') and $\beta$-actin (forward, 5'-ACCTTCTACAAT GAGCTGCG-3'; reverse, 5'-CCTGGATAGCAACGTACA TGG-3') were used.

Transfection of siRNAs. Specific siRNAs for silencing Smad3, the negative control siRNA (si-NC) and transfection kit were purchased from Ribobio (stQ0007005-1; Guangzhou, China). Cells were seeded in 24-well plate with the density of $10^{5} /$ well. After $24 \mathrm{~h}, 465.75 \mu \mathrm{l}$ cell culture medium, $30 \mu 1 \mathrm{1X}$ ribo $\mathrm{FECT}^{\mathrm{TM}}$ CP buffer, $1.25 \mu \mathrm{l}$ siRNA and $3 \mu \mathrm{l}$ ribo FECT $^{\text {TM }}$ CP reagent were added into each well. Cells were cultured in $5 \% \mathrm{CO}_{2}$ at $37^{\circ} \mathrm{C}$ for $48 \mathrm{~h}$ and then the expression of Smad3 was detected.

Xenograft tumor. Female immune-deficient BALB/C-nude mice (4 weeks old, $15-25 \mathrm{~g}$ body weight) were kept under controlled temperature and humidity with light-dark cycles in the animal room. A single cell suspension of $8 \times 10^{7}$ cells were subcutaneously inoculated into each mouse. The volume of tumors was calculated $\left(\mathrm{VT}=\right.$ length $\mathrm{x}$ width $\left.{ }^{2} \mathrm{x} 0.5\right)$ every other day from the tumor observation. The nude mice were euthanized and the xenograft tumors were harvested 12 days after implantation.

All animal experimental procedures were approved by Laboratory animal ethics committee of Capital Medical University (Beijing, China). The results of all animal experiments are reported in accordance with the ARRIVE guidelines.

Immunohistochemistry (IHC) staining. Human tumor samples from patients with breast cancer or BCBM were obtained from Cancer Hospital of Huanxing (Beijing, China). Tissues were harvested, fixed, paraffin-embedded, sectioned, and photographed. The sections were stained with hematoxylin. The expression of FSTL1 and Ki67 was determined using IHC staining. The primary antibody used was polyclonal goat anti- human FSTL1 antibody $(5 \mu \mathrm{g} / \mathrm{ml}$, AF1694; R\&D Systems) and rabbit anti-Ki67 antibody (1:250, ab16667; Abcam).

This study was approved by Cancer Hospital of Huanxing review board, and informed consent was obtained from all patients under the protocols prescribed by Cancer Hospital of Huanxing ethics committee. All procedures performed involving human participants were in accordance with the ethical standards of the Institutional and National Research Committee and with the 1964 Helsinki Declaration and its later amendments or comparable ethical standards.

Statistical analysis. Distributions of the data was tested for normality using D'Agostino and Pearson omnibus normality test. Statistically significant differences between two groups were determined by Student's t-test, and data are presented as the mean $\pm \mathrm{SD}$. The non-parametric Wilcoxon rank-sum test was used when the data were not normally distributed, and data are presented as median \pm the range. All statistical analyses were performed in GraphPad Prism 5 (GraphPad Software, San Diego, CA, USA), and $\mathrm{P}<0.05$ was considered statistically significant.

\section{Results}

Expression of FSTL1 in breast cancer cells and characterization of FSTL1 stable transfectants. The protein and mRNA levels of FSTL1 in four breast cancer cell lines MDA-MB-231, MCF7, ZR-75-1 and HCC38, and in the specific brain metastatic cell line 231-BR were measured (Fig. 1A). FSTL1 was hardly detected in the four breast cancer cell lines including MDA-MB-231, while it showed an elevated expression in the 231-BR (Fig. 1B). To investigate the functional role of FSTL1 in breast cancer cells, FSTL1 was overexpressed in the MDA-MB-231 cells using red fluorescence labeled lentivirus, and knocked down in 231-BR cells using Cherry fluorescence labeled lentivirus. More than $90 \%$ of MDA-MB-231 control cells (MDA-MB-231 ${ }^{\mathrm{CON}}$ ), FSTL1 overexpressed MDA-MB-231 cells (MDA-MB-231 ${ }^{\mathrm{FSTL} 1}$ ), 231-BR control cells $\left(231-\right.$ BR $\left.^{\text {sh CON }}\right)$ and FSTL1 knockdown 231-BR cells (231-BR ${ }^{\text {sh FSTLI}) ~ s h o w e d ~ e x p r e s s i o n ~ o f ~ R F P ~ o r ~ C h e r r y ~ f l u o r e s-~}$ cence, indicating a successful infection (Fig. 1C). The protein level of FSTL1 was obviously upregulated in MDA-MB-231 ${ }^{\text {FSTL1 }}$ cells compared with control (MDA-MB-231 ${ }^{\mathrm{CON}}$ ) (Fig. 1D, left panel), and downregulated in 231-BR ${ }^{\text {sh FSTL1 }}$ cells compared with control (231-BR $\left.{ }^{\mathrm{sh} C O N}\right)$ (Fig. 1D, right panel).

MDA-MB-231 cells show a higher proliferation rate than 231-BR cells. To compare the proliferation rates between MDA-MB-231 and 231-BR cells, MTT assay, RTCA and colony forming assay were performed. As shown in Fig. 2A, MDA-MB-231 cells grew faster compared with 231-BR cells as detected by MTT assay (3.65-fold on day 5, $\mathrm{P}<0.001$ ). Similar result was obtained in RTCA, that the cell growth of MDA-MB-231 was 3.9-fold faster than 231-BR on $96 \mathrm{~h}$ $(\mathrm{P}<0.001)$ (Fig. 2B). Furthermore, in colony forming assay, more colonies were observed in MDA-MB-231 cells compared with 231-BR (1.66-fold, $\mathrm{P}<0.01$ ) (Fig. $2 \mathrm{C}$ and $\mathrm{D}$ ).

In addition, apoptosis was investigated by measuring protein level of cleaved caspase-3 through western blotting. Results showed that the expression levels of cleaved caspase-3 in MDA-MB-231 and 231-BR cells were comparable, indicating that the inhibited cell growth in 231-BR cells was caused by suppressed cell proliferation (Fig. 2E).

FSTL1 overexpression inhibits cell proliferation of $M D A-M B-231$ and MCF7 cells in vitro. In order to elucidate the effect of FSTL1 overexpression on cell proliferation of MDA-MB-231 cells, MTT, RTCA and colony forming assay were carried out for the transfected MDA-MB-231 cells. After overexpression of FSTL1, the growth rate of MDA-MB-231 ${ }^{\text {FSTL1 }}$ was reduced by $53.21 \%$ $(\mathrm{P}<0.001)$ compared with MDA-MB-231 ${ }^{\mathrm{CON}}$ as detected by MTT assay on day 5 (Fig. 3A). Suppressed proliferation of 
A

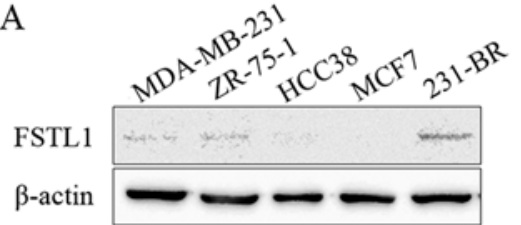

B
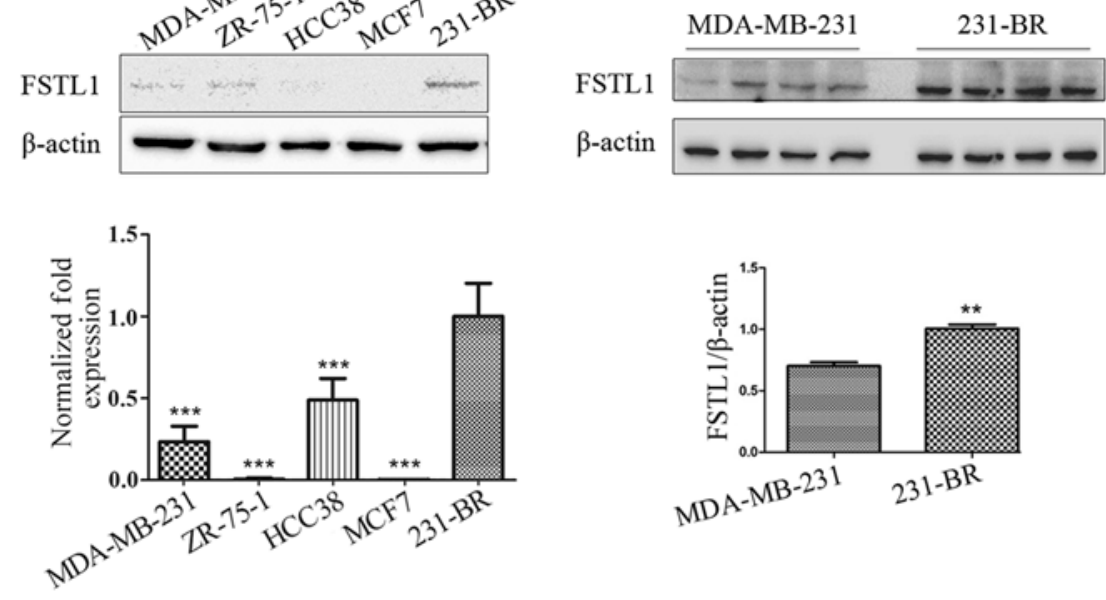

FSTLI



$M D A-M B-231 \quad 231-B R$

$\mathrm{D}$

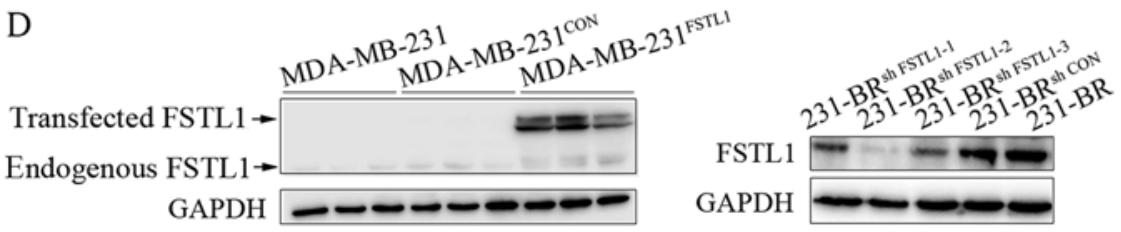

$\mathrm{C}$
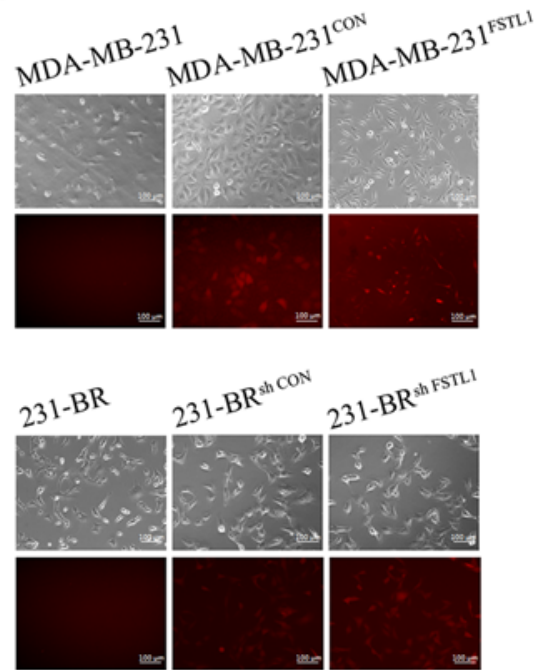

Figure 1. Expression of FSTL1 in breast cancer cells and characterization of FSTL1 stable transfectants. (A) The protein and mRNA levels of FSTL1 in MDA-MB-231, ZR-75-1, HCC38 and MCF7 cells were measured by western blotting (upper panel) and real-time PCR (lower panel). $\beta$-actin was used as internal control in the upper panel. The expression of first four cell lines were compared with that of 231-BR in the lower panel. (B) Relative expression levels of FSTL1 in MDA-MB-231 cells and 231-BR cells were detected by western blotting. $\beta$-actin was used as internal control. Values were expressed as mean $\pm \mathrm{SD}$ of results from at least three independent experiments. Statistical significant differences are marked $\left({ }^{* *} \mathrm{P}<0.01\right.$, unpaired two-tailed Student's $\mathrm{t}$-test and ${ }^{* * *} \mathrm{P}<0.001$, unpaired two-tailed Student's t-test). (C) MDA-MB-231 cells were infected with lentiviral vectors encoding FSTL1 (MDA-MB-231 $1^{\mathrm{FSTL}}$ ) or control vectors (MDA-MB-231 ${ }^{\mathrm{CON}}$ ). The infection was indicated by red fluorescent protein. 231-BR cells were infected with lentiviral vectors encoding FSTL1 specific shRNAs (231-BR ${ }^{\text {sh FSTLI }}$ ) or control vector $\left(231-\mathrm{BR}^{\text {sh CON }}\right.$ ). (D) The transfection efficiency of the two cell lines are evaluated using western blotting. FSTL1 (50 kDa)-RFP (26 kDa) fusion protein (76 kDa) was detected (upper panel). Three FSTL1 specific shRNA (shRNA-1, shRNA-2 and shRNA-3) were constructed, and the one with the highest efficiency (shRNA-2) was chosen for the following experiment (lower panel). GAPDH was used as an internal control.
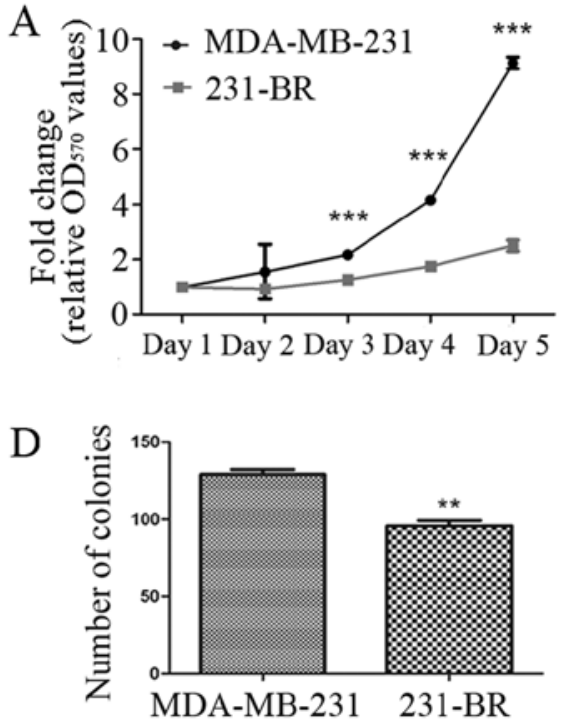

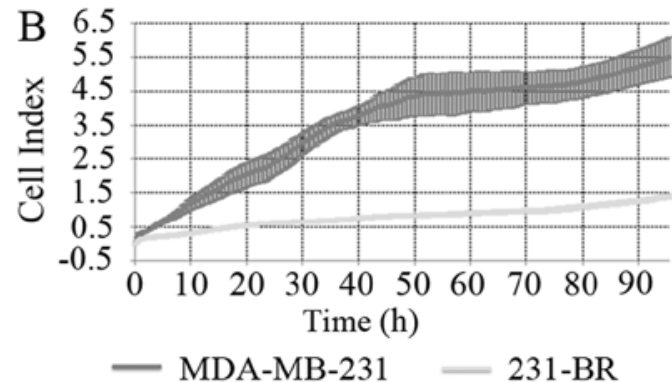

E

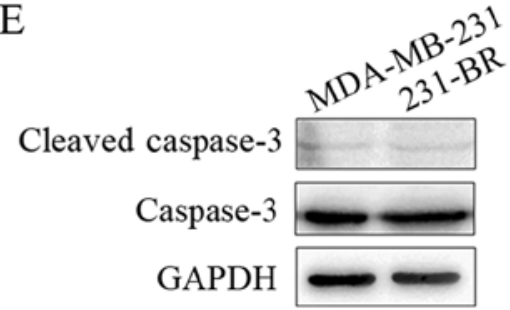

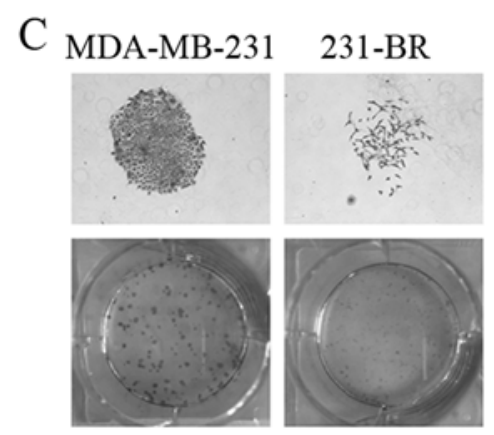

Figure 2. MDA-MB-231 shows a higher proliferation rate than 231-BR. (A and B) MDA-MB-231 and 231-BR cell proliferation was measured by MTT assay and RTCA, respectively. (C) The single colony and colonies of MDA-MB-231 and 231-BR cells were stained with crystal violet. (D) Colony numbers were counted. (E) The protein level of cleaved caspase-3 was measured by western blotting. GAPDH was used as an internal control. Values in bar graphs represent mean \pm SD of results from at least three independent experiments, statistical significant differences are marked $\left({ }^{* *} \mathrm{P}<0.01 ;{ }^{* * *} \mathrm{P}<0.001\right.$, unpaired two-tailed Student's t-test).

MDA-MB-231 ${ }^{\mathrm{FSTL} 1}$ cells was also detected in RTCA $(42.29 \%$, $\mathrm{P}<0.001$ ) (Fig. 3B). In addition, in colony forming assay, the number of MDA-MB-231 $1^{\text {FSTL1 }}$ cell colonies was reduced by $51.29 \%$ compared with control $(\mathrm{P}<0.001)$ (Fig. 3C and D). 

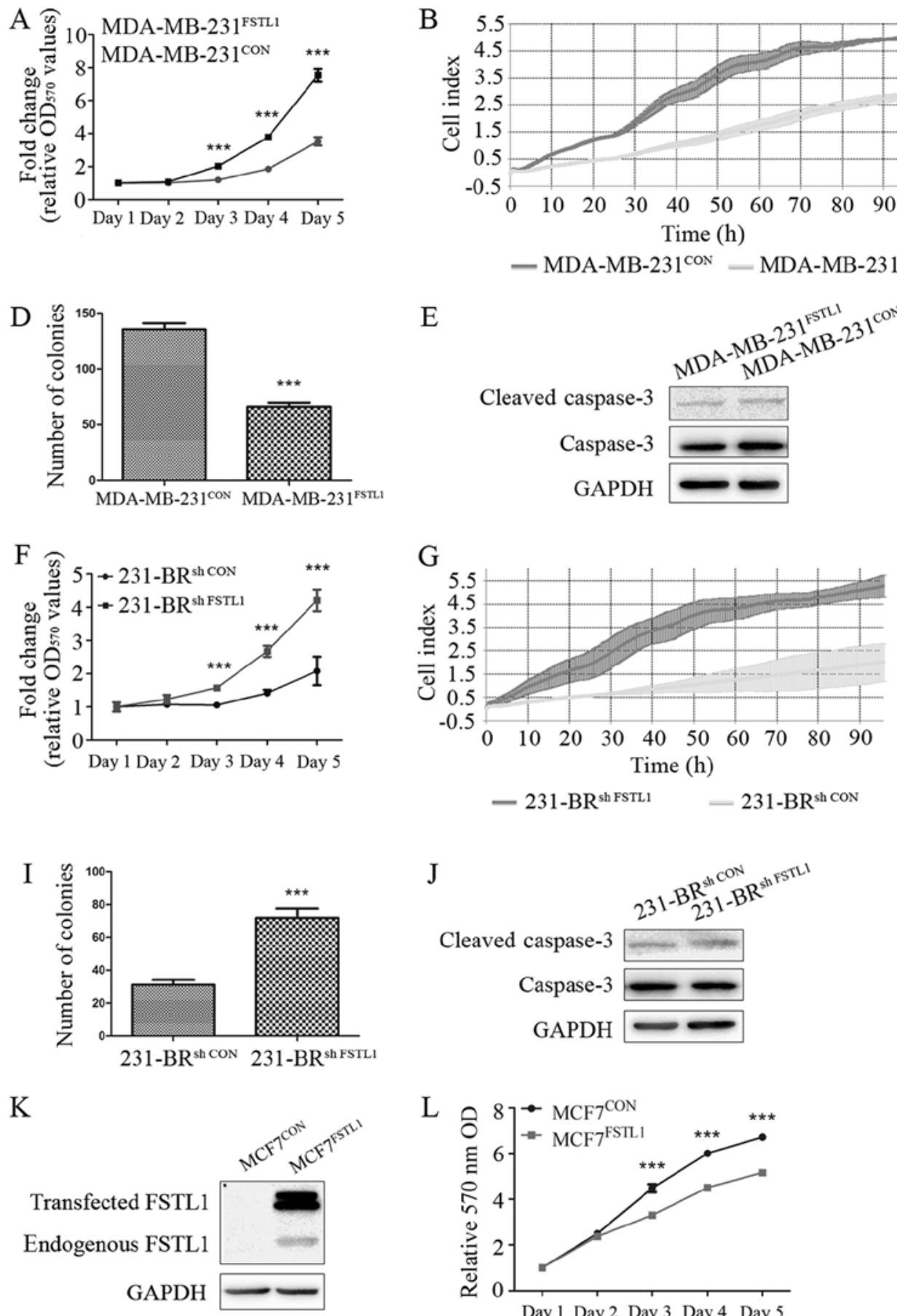

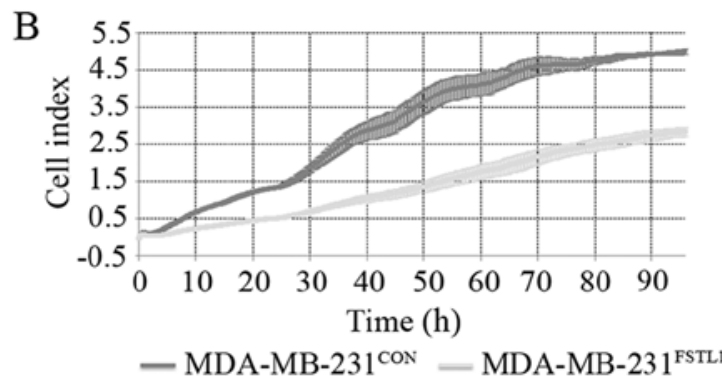

$\mathrm{E}$


$\mathrm{H}$
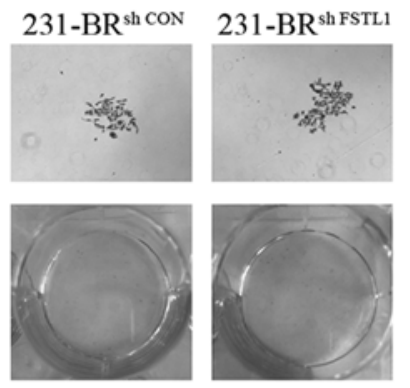

$\mathrm{J}$

$$
-231-\mathrm{BR}^{\text {sh FSTL1 }}-231-\mathrm{BR}^{\text {sh } \mathrm{CON}}
$$
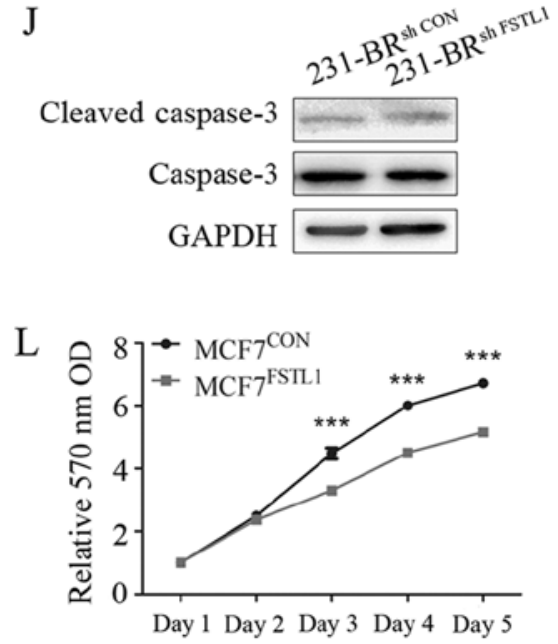

$\mathrm{M}$

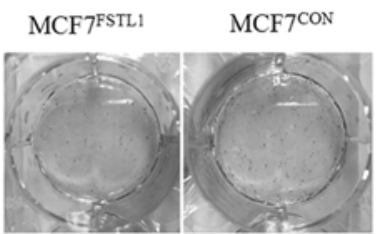

$\mathrm{N}$

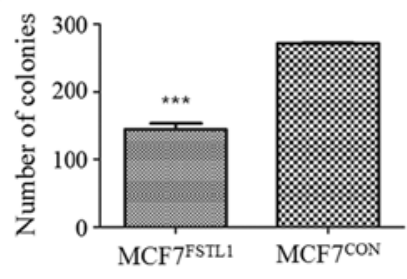

Figure 3. Effects of FSTL1 on cell proliferation. (A and B) MDA-MB-231 $1^{\mathrm{FSTL} 1}$ and MDA-MB-231 ${ }^{\mathrm{CON}}$ cell proliferation were measured by MTT assay and RTCA. (C) Cell proliferation was measured by colony forming assay, and the colonies were stained with crystal violet. (D) The colony numbers were counted. (E) To identify the apoptosis of MDA-MB-231 $1^{\mathrm{FTL}}$ and MDA-MB-231 $1^{\mathrm{CON}}$ cells, the protein level of cleaved caspase-3 was measured by western blotting, and GAPDH was used as an internal control. (F and G) $231-\mathrm{BR}^{\text {sh FSTL1 }}$ and $231-\mathrm{BR}^{\text {sh CON }}$ cell proliferation was measured by MTT assay and RTCA. (H) The colonies of 231-BR ${ }^{\text {sh FSTL }}$ and 231-BR ${ }^{\text {sh CON }}$ were stained with crystal violet. (I) The colony numbers were counted. (J) The protein level of cleaved caspase-3 was measured by western blotting to examine apoptosis of $231-\mathrm{BR}^{\text {sh FSTL1 }}$ and $231-\mathrm{BR}^{\text {sh CON }}$ cells, and GAPDH was used as an internal control. (K) MCF7 cells were infected with lentiviral vectors encoding FSTL1 (MCF7 ${ }^{\mathrm{FSTL}}$ ) or control (MCF7 ${ }^{\mathrm{CON}}$ ), and FSTL1 was detected by western blotting. (L) The proliferation of MCF $7^{\mathrm{FSTL}}$ and MCF7 $7^{\mathrm{CON}}$ were measured by MTT assay. (M) The proliferation of MCF7 $7^{\mathrm{FSTL} 1}$ and MCF7 $7^{\mathrm{CON}}$ were measured by colony forming assay, and the colonies were stained with crystal violet. $(\mathrm{N})$ The colony numbers were counted. The data are shown as mean $\pm \mathrm{SD}$ of at least three independent experiments $\left({ }^{* *} \mathrm{P}<0.01 ;{ }^{* * *} \mathrm{P}<0.001\right.$, unpaired two-tailed Student's t-test).

The protein level of cleaved caspase-3 was measured to detect cell apoptosis, and no significant difference was observed (Fig. 3E). To validate the effect of FSTL1 on breast cancer cell proliferation, FSTL1 was also overexpressed in MCF7 cells (Fig. 3K). Cell proliferation was evaluated by
MTT and colony forming assay and similar results were observed (Fig. 3L-N).

FSTL1 knockdown promotes cell proliferation of 231-BR cells in vitro. MTT, RTCA and colony forming assay were 


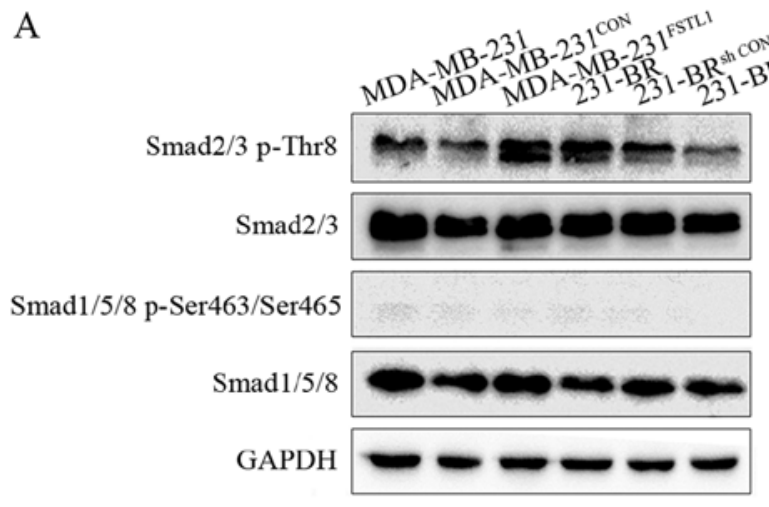

B

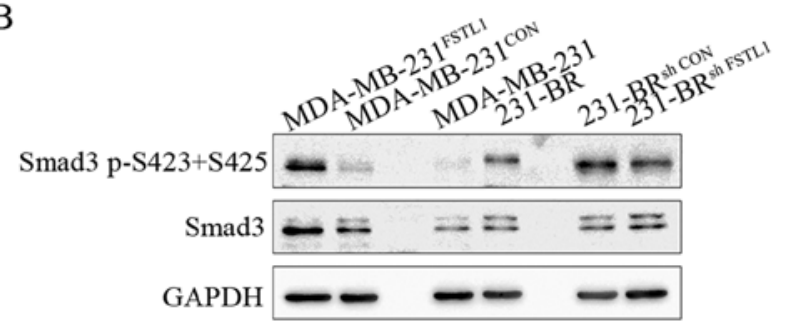

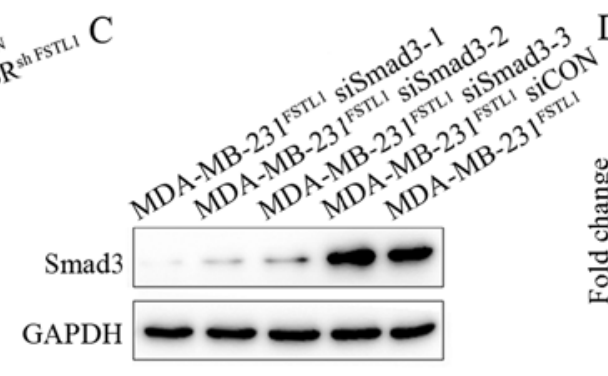

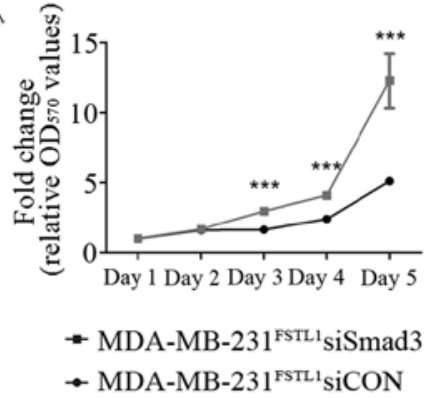

$\mathrm{E}$

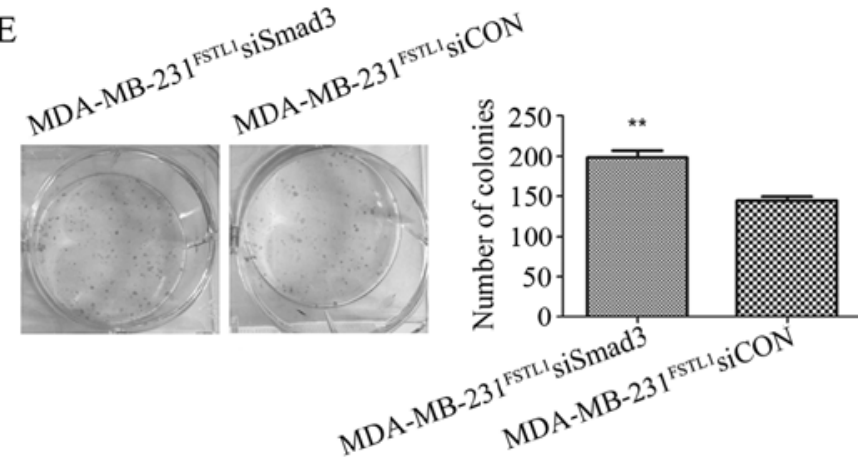

Figure 4. FSTL1 regulates the proliferation of MDA-MB-231 and 231-BR cells through TGF- $\beta 1$-Smad2/3 signaling pathway. (A) The protein level of Smad2/3, phosphorylated-Smad2/3, Smad1/5/8 and phosphorylated-Smad1/5/8 were measured by western blotting. GAPDH was used as an internal control. (B) The protein levels of Smad3 and phosphorylated-Smad3 (S423+S425) were measured by western blotting. GAPDH was used as an internal control. (C) The protein levels of Smad3 in MDA-MB-231 ${ }^{\text {FSTL1 }}$ cells treated with different siSmad3 were measured by western blotting. GAPDH was used as internal control. MDA-MB-231 ${ }^{\text {FSTL1 }}$ siSmad3 had the highest silence efficiency (MDA-MB-231 ${ }^{\text {FSTL1 }}$ siSmad3-1) was chosen for the following experiment. (D) The proliferation of MDA-MB-231 ${ }^{\text {FSTL1 }}$ cells treated with siSmad3 was measured by MTT assay. (E) The colonies of MDA-MB-231 ${ }^{\text {FSTL1 }}$ siSmad3 and MDA-MB-231 ${ }^{\text {FSTL1 }}$ siCON cells were stained with crystal violet and the number of colonies was counted. The data are shown as the mean \pm SD of results from at least three independent experiments $\left({ }^{* *} \mathrm{P}<0.01 ;{ }^{* * *} \mathrm{P}<0.001\right.$, unpaired two-tailed Student's t-test).

also carried out for the transfected 231-BR cells to explore the effect of FSTL1 knockdown on cell proliferation in 231-BR cells. As expected, the knockdown of FSTL1 in 231-BR cells increased cell proliferation by 1.67 -fold on day $5(\mathrm{P}<0.001)$, as detected by MTT assay (Fig. 3F). Consistently, RTCA showed that the growth of 231-BR ${ }^{\text {sh FSTL1 }}$ was 2.63 -fold $(\mathrm{P}<0.001)$ faster than 231-BR ${ }^{\text {sh CON }}$ (Fig. 3G). In addition, knockdown of FSTL1 in 231-BR cells increased colony size and number in colony forming assay (1.98-fold, $\mathrm{P}<0.001)$ (Fig. $3 \mathrm{H}$ and I). Expression level of cleaved caspase- 3 was also detected in these transfected cell lines, and no significant difference was observed (Fig. 3J).

FSTL1 inhibits the proliferation of MDA-MB-231 and 231-BR cells by regulating TGF- $\beta 1$ signaling pathway through Smad3. TGF- $\beta$ superfamily signaling pathway plays critical roles in the regulation of cancer cell growth (31). In addition, FSTL1 has been showed to activate TGF- $\beta 1-\mathrm{Smad} 2 / 3$ signaling pathway in pulmonary fibrogenesis (32) and myocardium (22), and inhibit BMP-Smad1/5/8 signaling pathway in controlling mouse lung development (33). These observations motivated us to question if there is an interaction between FSTL1 and TGF- $\beta$ signaling pathways that may contribute to the modification of cell proliferation.

As shown in Fig. 4A, lower phosphorylation of $\mathrm{Smad} 2 / 3$ was detected in MDA-MB-231 cells than 231-BR cells. In the transfected cell lines, the phosphorylation of $\operatorname{Smad} 2 / 3$ was increased after FSTL1 overexpression in MDA-MB-231 $1^{\text {FSTL1 }}$ cells compared with MDA-MB-231 ${ }^{\mathrm{CON}}$ (Fig. 4A), and decreased

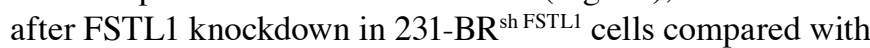
231-BR ${ }^{\text {sh CON }}$ cells (Fig. 4A). The phosphorylated Smad1/5/8 was hardly detected in these cell lines, suggesting an inactivation of BMP-Smad1/5/8 signaling pathway (Fig. 4A). These observations indicated that FSTL1 plays a role in the regulation of TGF- $\beta 1-\mathrm{Smad} 2 / 3$ signaling pathway, but not BMP-Smad1/5/8, in MDA-MB-231 and 231-BR cells.

Considering that $\mathrm{Smad} 3$ plays a central role in TGF- $\beta$ signaling pathway, we performed further studies to test if FSTL1 regulated cell proliferation through the TGF- $\beta$ pathway by regulating Smad3. As shown in Fig. 4B, phosphorylation level of Smad3 was higher in 231-BR cells than MDA-MB-231 cells. After transfection, the phosphorylation of Smad3 was increased after FSTL1 overexpression (in MDA-MB-231 $1^{\text {FSTL1 }}$ cells) and decreased after FSTL1 knockdown (in 231-BR ${ }^{\text {sh FSTL1 }}$ cells), compared with their corresponding controls. Next, Smad3 was knocked down by siRNAs in MDA-MB-231 $1^{\text {FSTL1 }}$ cells. Expression levels of Smad3 were measured using western blotting and the siRNA with highest silence efficiency (siSmad3-1) was used for the following experiments (Fig. 4C). As evaluated by MTT assay, knockdown of Smad3 in MDA-MB-231 ${ }^{\mathrm{FSTL} 1}$ significantly enhanced cell proliferation by 2.93-fold $(\mathrm{P}<0.001)$ on day 5 compare with control (Fig. 4D). Colony forming assay was also carried out, showing that colony number of MDA-MB-231 ${ }^{\text {FSTL1 }}$ cells was 1.37 -fold 
A

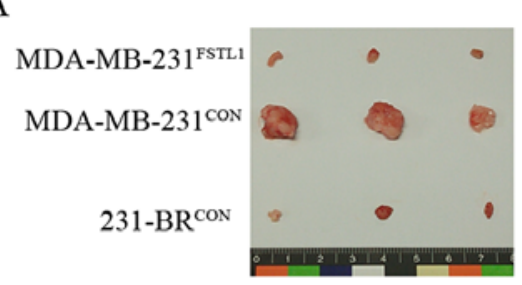

B

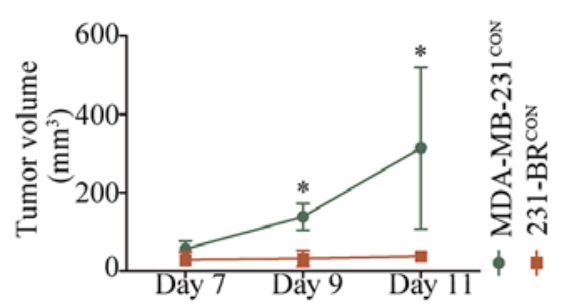

C

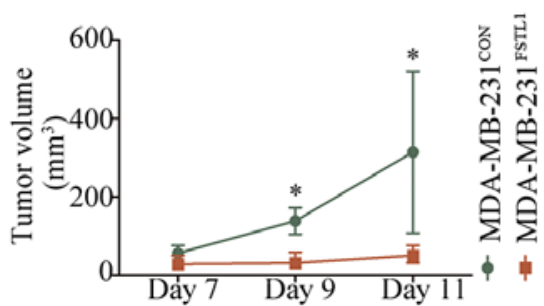

Figure 5. FSTL1 overexpression inhibits the proliferation of MDA-MB-231 cells in vivo. (A) MDA-MB-231 ${ }^{\mathrm{FSTL} 1}$, MDA-MB-231 ${ }^{\mathrm{CON}}$ and 231-BR ${ }^{\mathrm{CON}}$ cells were injected into the nude mice $(\mathrm{n}=5)$, the tumors were harvested and photographed after 12 days. (B and C) The volume of tumors was measured 7 days after cell inoculation. The data are presented as the median \pm the range $(\mathrm{n}=5)\left({ }^{*} \mathrm{P}<0.05\right.$ using a non-parametric Wilcoxon rank-sum test).
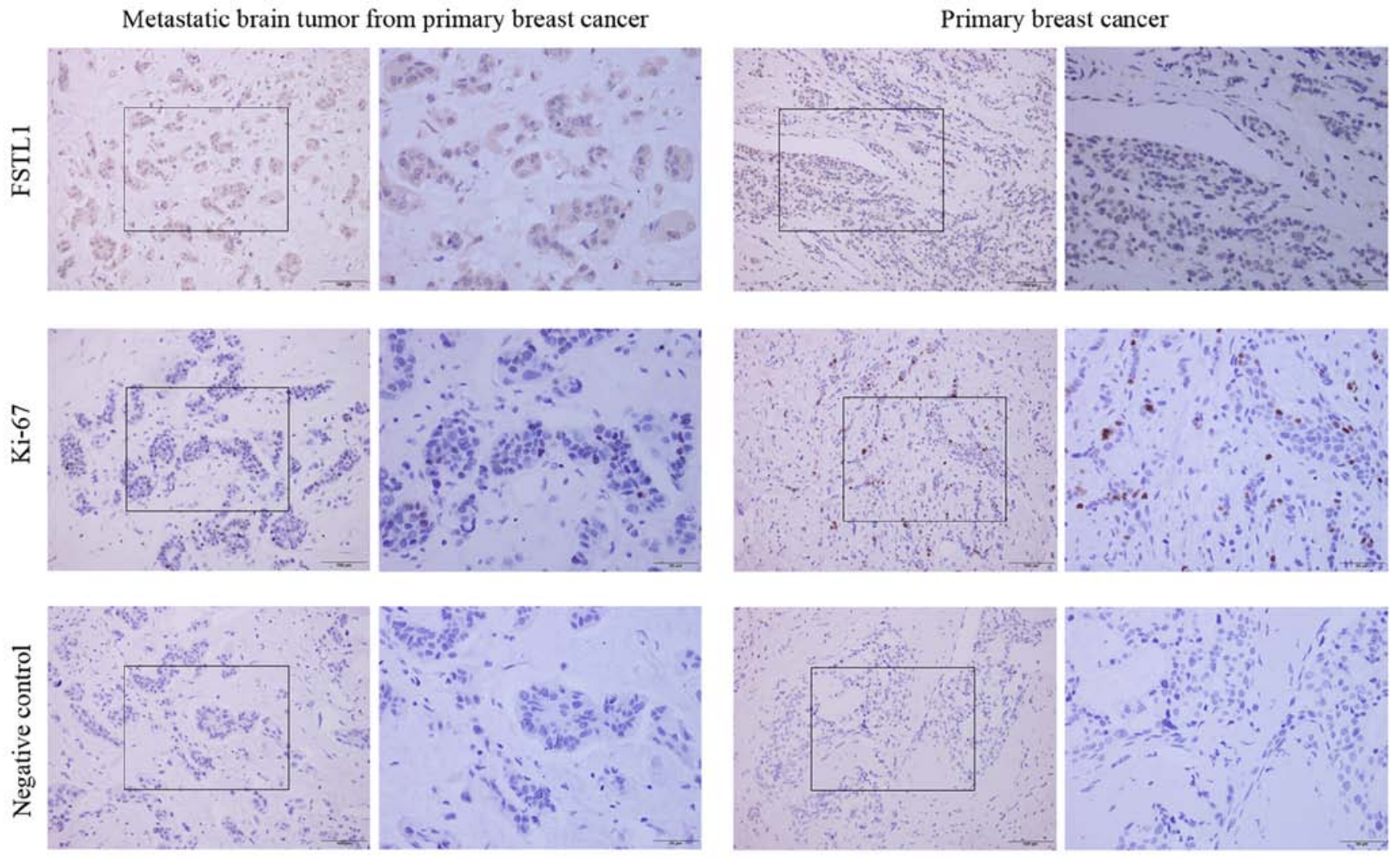

Figure 6. Immunostaining of human specimens. Expression of FSTL1 and Ki67 was detected in breast cancer specimens and metastatic brain tumor from primary breast cancer, and representative slides are shown. Scale bar, $100 \mu \mathrm{m}$ (left) and $50 \mu \mathrm{m}$ (right).

increased $(\mathrm{P}<0.01)$ after Smad3 knockdown (Fig. 4E). These results revealed that knockdown of Smad3 restored the cell proliferation, which was inhibited by FSTL1 overexpression in MDA-MB-231 ${ }^{\text {FSTL1 }}$ cells.

FSTL1 overexpression inhibits the proliferation of $M D A-M B-231$ cells in vivo. To investigate the effect of FSTL1 on tumor growth in vivo, MDA-MB-231 ${ }^{\mathrm{FSTL}}$, MDA-MB-231 ${ }^{\mathrm{CON}}$ and $231-\mathrm{BR}^{\mathrm{CON}}$ cells were inoculated subcutaneously into female nude mice. Tumor volume was measured every other day from one week after cell injection. Tumors were harvested and photographed on day 12 (Fig. 5A). Results showed that the tumor volume in 231-BR ${ }^{\mathrm{CON}}$ cell-bearing mice was significantly smaller than the xenografts formed by MDA-MB-231 $1^{\mathrm{CON}}$ cells $(\mathrm{n}=5, \mathrm{P}<0.05)$ (Fig. 5B), and reduction in tumor volume could be detected in MDA-MB-231 $1^{\mathrm{FSTL} 1}$ group compared with the control group $(n=5, P<0.05)$ (Fig. $5 C$ ). These results indicated that MDA-MB-231 had a higher proliferation rate than 231-BR cells and FSTL1 overexpression inhibited the proliferation of MDA-MB-231 in vivo, which was in agreement with the study in vitro.

One metastatic brain tumor from primary breast cancer of BCBM patient showed a high-level of FSTL1, while low-level of this protein was detected in primary breast cancer specimens. We wondered whether the correlation of FSTL1 and proliferation observed in vitro and in vivo also existed in human specimens, the FSTL1 expression was detected in primary breast cancer specimens $(n=5)$ and one metastatic brain tumor $(n=1)$ from primary breast cancer. As IHC staining demonstrated (Fig. 6), the resected metastatic brain tumor from primary breast cancer showed a higher expression level of FSTL1; whereas the Ki67 expression was lower than that in primary breast cancer. On the other hand, the five specimens 
of primary breast cancer all showed a lower expression level of FSTL1 and a higher expression of Ki67 (Fig. 6). This result showed a trend similar to the results in vitro and in vivo.

The collection of brain metastatic tumors from BCBM patients is difficult because only a small part of BCBM patients with a single brain metastasis will undergo neurosurgical resection. In this study, only one metastatic brain tumor from primary breast cancer was obtained so that this result only showed a trend which may not be significant. More evidence is required to confirm this result.

\section{Discussion}

Aberrant expression and various functions of FSTL1 have been observed in tumor cell lines and clinical tumor tissues, suggesting a role of FSTL1 in cancer. However, the underlying mechanisms have rarely been studied and remain largely unknown. In some studies, FSTL1 was identified as a tumorsuppressive protein. It was found to be downregulated in metastatic clear-cell renal-cell carcinoma and considered to be related to metastasis (34). In ovarian and endometrial carcinogenesis, downregulation of FSTL1 was detected and a tumor suppressor role was demonstrated (25). In contrast, FSTL1 overexpression was observed in glioblastoma as a hallmark of poor prognosis by Reddy et al in 2010 (23). In that study, analysis of retrospective GBM cases with known survival data revealed that the coexpression of FSTL1 with p53 was associated with poor survival. However, the functional role of FSTL1 in astrocytomas was not investigated, and the signaling pathways involved in the expression of FSTL1 remain to be determined (23). To date, the functional roles of FSTL1 in cancer remain controversial and unclear. The role of FSTL1 in breast cancer or BCBM has not been investigated. Our study is the first report documenting the increased level of FSTL1 in 231-BR cell line and linking the possible functions of FSTL1 in breast cancer progression.

The signaling pathways of FSTL1 involved in cancer are poorly defined. The following lines of evidence motivated us to investigate the correlation between FSTL1 and TGF- $\beta$ signaling pathway in breast cancer cells. Firstly, as a TGF- $\beta 1$ inducible gene, FSTL1 encodes a secreted glycoprotein belonging to a group of matricellular proteins (19). Two recent studies showed that it activated TGF- $\beta 1-S m a d 2 / 3$ signaling in pulmonary fibrogenesis (32) and myocardium (22), respectively. Also, in lung development, it can reduce the activity of TGF- $\beta$ /BMP signaling (33). These studies indicated a role of FSTL1 in regulating TGF- $\beta$ signaling pathways. Secondly, as a pleiotropic cytokine, TGF- $\beta$ signaling pathways regulates diverse cellular processes in cancer, including apoptosis, cell growth and epithelial-mesenchymal transition (35). A direct influence on breast cancer pathophysiology by TGF- $\beta 1$ was documented (18). It inhibits breast cancer cell growth and promotes apoptosis in early stages, while it is related to increased tumor progression in late stages. Moreover, TGF- $\beta 1$ has already been shown to inhibit the anchorageindependent growth of MDA-MB-231 and 231-BR (9). Taken these two points into consideration, we investigated the role of FSTL1 in the regulation of TGF- $\beta$ signaling pathways. We detected Smad2/3-mediated TGF- $\beta 1$ and Smad1/5/8-mediated TGF- $\beta$ /BMP signaling pathway in wild-type and transfected
MDA-MB-231 and 231-BR cells. We clarified that the TGF- $\beta 1$ Smad $2 / 3$ signaling pathway was, at least partly, the molecular mechanism whereby FSTL1 modulates the cell proliferation rate. To date, it is still not clear if FSTL1 exerts its effects in autocrine and paracrine manner as a secreted protein in cancer. In 2010, DIP2A was suggested to be a potential receptor of FSTL1 that mediates the protective roles of cardiomyocytes, while the signaling pathways involved in this process were not clarified $(36,37)$. In addition, the roles of DIP2A in cancers have not been investigated yet. Therefore, to detect the expression of DIP2A in breast cancer cells and patient tissues, and to find out the signaling pathways involved may also help to study the effects of FSTL1 on breast cancer cell proliferation. This will be investigated in a future study.

The metastatic cascade of BCBM involves a series of well-defined steps including local invasion, intravasation, survival in the circulation, extravasation, colonization and proliferation, while the mechanisms underlying this complex process are largely unknown. To date, majority of the preclinical studies focuses on early stages of BCBM, to find the possible risk factors for the development of brain metastases. However, the present study may provide another view and demonstrate a likely role of FSTL1 in the step of 'proliferation', which is the last step of BCBM cells after they entered the brain. However, the effects of brain microenvironment and its mechanical properties on breast cancer cells should also be investigated.

Although FSTL1 showed an anti-proliferative effect on breast cancer cells, it is too early to say if it is an antitumor factor, and there is still a long way to go to clarify the role of FSTL1 in breast cancer prognosis and to design drug for breast cancer patient. In the present study, FSTL1 was suggested to exert its effect through regulating TGF- $\beta$ signaling, which could inhibit cell growth in early stages and increased tumor progression in late stages and has 'two faces' in cancer (18). Also, added to the inhibitory effect of FSTL1 on cell proliferation and the overexpression of FSTL1 in metastatic 231-BR cells observed in the present study, FSTL1 may have dynamic roles in breast cancer. The effects of FSTL1 on cell invasion, and cell migration, should also be taken into consideration, which may affect the tumor malignant process. Additionally, the association of FSTL1 level with BCBM clinical prognosis needs to be evaluated. With the rapid development of the molecular biology techniques, the use of genetic screens that delineate the tumor-suppressive versus the possible tumorpromoting roles of FSTL1 may provide a basis for new study that will enable the targeting of its specific oncogenic subarms. At present, studies focusing on the effect of FSTL1 on invasion and metastasis of breast cancer cells are undergoing in our laboratory.

As the expression of FSTL1 in clinical biopsy specimens of breast cancer patients has rarely been documented, we tried to detect the expression of FSTL1 in human tissue. However, patients with BCBM often bear multiple brain metastases that are not suitable to undergo surgical resection. Moreover, the whole brain radiation remains standard in the management of BCBM. Only for a small part of BCBM patients with a single brain metastasis, neurosurgical resection will be carried out followed by WBR (38). It makes the collection of brain metastatic tumors from BCBM patients difficult. In this study, only one metastatic brain tumor from 
primary breast cancer was obtained (Fig. 6) from a patient with a single brain metastasis, and a higher expression level of FSTL1 was observed $(n=1)$. Meanwhile, the expression of FSTL1 was much lower in breast cancer in situ $(n=5)$. More evidence is required from clinical data, and the specimens are being collected continually.

In the present study, a high-level expression of FSTL1 was first detected in the brain metastatic breast cancer cell line 231-BR. An inhibitory effect of FSTL1 on cell proliferation was determined in 231-BR and MDA-MB-231 cells in vitro and in vivo. This anti-proliferative effect was further explored to be associated with regulation of TGF- $\beta$ signaling pathway through Smad3. Our data define FSTL1 as an inhibitor of proliferation of breast cancer cells, which may provide new insight into the mechanism underlying BCBM.

\section{Acknowledgements}

We thank Dr Patricia S. Steeg (National Cancer Institute, Bethesda, MD, USA) for the 231-BR cell line, Dr Jun Wan (Hong Kong University of Science and Technology, Hong Kong, China) for the MDA-MB-231 and MCF7 cell lines, and Dr Haili Qian (Cancer Hospital Chinese Academy of Medical Sciences, Beijing, China) for the ZR-75-1 and HCC38 cell lines. This study was supported by National Natural Science Foundation of China, grant no. 81602532; Beijing Natural Science Foundation, grant nos. 7164238 and 7152016; Beijing Municipal Commission of Education, grant no. KM201410025002; Beijing Municipal Organization Department Talents Project, grant no. 2015000020124G113; Support Project of High-level Teachers in Beijing Municipal Universities in the Period of 13th Five-year Plan, grant no. IDHT20170516.

\section{References}

1. Siegel RL, Miller KD and Jemal A: Cancer statistics, 2016. CA Cancer J Clin 66: 7-30, 2016.

2. Kienast Y, von Baumgarten L, Fuhrmann M, Klinkert WE, Goldbrunner R, Herms J and Winkler F: Real-time imaging reveals the single steps of brain metastasis formation. Nat Med 16: 116-122, 2010.

3. Palmieri D, Smith QR, Lockman PR, Bronder J, Gril B, Chambers AF, Weil RJ and Steeg PS: Brain metastases of breast cancer. Breast Dis 26: 139-147, 2006-2007.

4. Kodack DP, Askoxylakis V, Ferraro GB, Fukumura D and Jain RK: Emerging strategies for treating brain metastases from breast cancer. Cancer Cell 27: 163-175, 2015.

5. Rostami R, Mittal S, Rostami P, Tavassoli F and Jabbari B: Brain metastasis in breast cancer: A comprehensive literature review. J Neurooncol 127: 407-414, 2016.

6. Sihto H, Lundin J, Lundin M, Lehtimäki T, Ristimäki A, Holli K, Sailas L, Kataja V, Turpeenniemi-Hujanen T, Isola J, et al: Breast cancer biological subtypes and protein expression predict for the preferential distant metastasis sites: A nationwide cohort study. Breast Cancer Res 13: R87, 2011.

7. Gil-Gil MJ, Martinez-Garcia M, Sierra A, Conesa G, Del Barco S, González-Jimenez S and Villà S: Breast cancer brain metastases: A review of the literature and a current multidisciplinary management guideline. Clin Transl Oncol 16: 436-446, 2014.

8. Lam SW, Jimenez CR and Boven E: Breast cancer classification by proteomic technologies: Current state of knowledge. Cancer Treat Rev 40: 129-138, 2014.

9. Yoneda T, Williams PJ, Hiraga T, Niewolna M and Nishimura R: A bone-seeking clone exhibits different biological properties from the MDA-MB-231 parental human breast cancer cells and a brain-seeking clone in vivo and in vitro. J Bone Miner Res 16: 1486-1495, 2001
10. Stark AM, Anuszkiewicz B, Mentlein R, Yoneda T, Mehdorn HM and Held-Feindt J: Differential expression of matrix metalloproteinases in brain- and bone-seeking clones of metastatic MDA-MB-231 breast cancer cells. J Neurooncol 81: 39-48, 2007.

11. Dun MD, Chalkley RJ, Faulkner S, Keene S, Avery-Kiejda KA, Scott RJ, Falkenby LG, Cairns MJ, Larsen MR, Bradshaw RA, et al: Proteotranscriptomic profiling of 231-BR breast cancer cells: Identification of potential biomarkers and therapeutic targets for brain metastasis. Mol Cell Proteomics 14: 2316-2330, 2015.

12. Fitzgerald DP, Subramanian P, Deshpande M, Graves C, Gordon I, Qian Y, Snitkovsky Y, Liewehr DJ, Steinberg SM, Paltán-Ortiz JD, et al: Opposing effects of pigment epitheliumderived factor on breast cancer cell versus neuronal survival: Implication for brain metastasis and metastasis-induced brain damage. Cancer Res 72: 144-153, 2012.

13. Gril B, Palmieri D, Bronder JL, Herring JM, Vega-Valle E, Feigenbaum L, Liewehr DJ, Steinberg SM, Merino MJ, Rubin SD, et al: Effect of lapatinib on the outgrowth of metastatic breast cancer cells to the brain. J Natl Cancer Inst 100: 1092-1103, 2008.

14. Lockman PR, Mittapalli RK, Taskar KS, Rudraraju V, Gril B, Bohn KA, Adkins CE, Roberts A, Thorsheim HR, Gaasch JA, et al: Heterogeneous blood-tumor barrier permeability determines drug efficacy in experimental brain metastases of breast cancer. Clin Cancer Res 16: 5664-5678, 2010.

15. Palmieri D, Bronder JL, Herring JM, Yoneda T, Weil RJ,Stark AM, Kurek R, Vega-Valle E, Feigenbaum L, Halverson D, et al: Her-2 overexpression increases the metastatic outgrowth of breast cancer cells in the brain. Cancer Res 67: 4190-4198, 2007.

16. Percy DB, Ribot EJ, Chen Y, McFadden C, Simedrea C, Steeg PS, Chambers AF and Foster PJ: In vivo characterization of changing blood-tumor barrier permeability in a mouse model of breast cancer metastasis: A complementary magnetic resonance imaging approach. Invest Radiol 46: 718-725, 2011.

17. Chen $G$ and Davies MA: Emerging insights into the molecular biology of brain metastases. Biochem Pharmacol 83: 305-314, 2012.

18. Zarzynska JM: Two faces of TGF-beta1 in breast cancer. Mediators Inflamm 2014: 141747, 2014.

19. Shibanuma M, Mashimo J, Mita A, Kuroki T and Nose K: Cloning from a mouse osteoblastic cell line of a set of transforminggrowth-factor-beta 1-regulated genes, one of which seems to encode a follistatin-related polypeptide. Eur J Biochem 217: 13-19, 1993

20. Adams D, Larman B and Oxburgh L: Developmental expression of mouse Follistatin-like 1 (Fst11): Dynamic regulation during organogenesis of the kidney and lung. Gene Expr Patterns 7: 491-500, 2007.

21. Kawabata D, Tanaka M, Fujii T, Umehara H, Fujita Y, Yoshifuji H, Mimori T and Ozaki S: Ameliorative effects of follistatin-related protein/TSC-36/FSTL1 on joint inflammation in a mouse model of arthritis. Arthritis Rheum 50: 660-668, 2004.

22. Xi Y, Gong DW and Tian Z: FSTL1 as a potential mediator of exercise-induced cardioprotection in post-myocardial infarction rats. Sci Rep 6: 32424, 2016.

23. Reddy SP, Britto R, Vinnakota K, Aparna H, Sreepathi HK, Thota B, Kumari A, Shilpa BM, Vrinda M, Umesh S, et al: Novel glioblastoma markers with diagnostic and prognostic value identified through transcriptome analysis. Clin Cancer Res 14: 2978-2987, 2008.

24. Kudo-Saito C, Fuwa T, Murakami K and Kawakami Y: Targeting FSTL1 prevents tumor bone metastasis and consequent immune dysfunction. Cancer Res 73: 6185-6193, 2013.

25. Chan QK, Ngan HY, Ip PP, Liu VW, Xue WC and Cheung AN: Tumor suppressor effect of follistatin-like 1 in ovarian and endometrial carcinogenesis: A differential expression and functional analysis. Carcinogenesis 30: 114-121, 2009.

26. Chaly Y, Hostager B, Smith S and Hirsch R: Follistatin-like protein 1 and its role in inflammation and inflammatory diseases. Immunol Res 59: 266-272, 2014.

27. Chen SX, Xu XE, Wang XQ, Cui SJ, Xu LL, Jiang YH, Zhang Y, Yan HB, Zhang Q, Qiao J, et al: Identification of colonic fibroblast secretomes reveals secretory factors regulating colon cancer cell proliferation. J Proteomics 110: 155-171, 2014.

28. Bae K, Park KE, Han J, Kim J, Kim K and Yoon KA: Mitotic cell death caused by follistatin-like 1 inhibition is associated with up-regulated Bim by inactivated Erk1/2 in human lung cancer cells. Oncotarget 7: 18076-18084, 2016. 
29. Tan J, Yang S, Shen P, Sun H, Xiao J, Wang Y, Wu B, Ji F, Yan J, Xue $\mathrm{H}$, et al: $\mathrm{C}$-kit signaling promotes proliferation and invasion of colorectal mucinous adenocarcinoma in a murine model. Oncotarget 6: 27037-27048, 2015.

30. Meng X, Zheng R, Zhang Y, Qiao M, Liu L, Jing P, Wang L, Liu J and Gao Y: An activated sympathetic nervous system affects white adipocyte differentiation and lipolysis in a rat model of Parkinson's disease. J Neurosci Res 93: 350-360, 2015.

31. Wieser R: The transforming growth factor-beta signaling pathway in tumorigenesis. Curr Opin Oncol 13: 70-77, 2001.

32. Dong Y, Geng Y, Li L, Li X, Yan X, Fang Y, Li X, Dong S, Liu X, Li X, et al: Blocking follistatin-like 1 attenuates bleomycin-induced pulmonary fibrosis in mice. J Exp Med 212: 235-252, 2015.

33. Geng Y, Dong Y, Yu M, Zhang L, Yan X, Sun J, Qiao L, Geng H, Nakajima M, Furuichi T, et al: Follistatin-like 1 (Fstl1) is a bone morphogenetic protein (BMP) 4 signaling antagonis in controlling mouse lung development. Proc Natl Acad Sci USA 108: 7058-7063, 2011.
34. Tan X, Zhai Y, Chang W, Hou J, He S, Lin L, Yu Y, Xu D, Xiao J, Ma L, et al: Global analysis of metastasis-associated gene expression in primary cultures from clinical specimens of clear-cell renal-cell carcinoma. Int J Cancer 123: 1080-1088, 2008. 35. Massagué J: TGFbeta in cancer. Cell 134: 215-230, 2008.

36. Tanaka M, Murakami K, Ozaki S, Imura Y, Tong XP, Watanabe T, Sawaki T, Kawanami T, Kawabata D, Fujii T, et al: DIP2 discointeracting protein 2 homolog A (Drosophila) is a candidate receptor for follistatin-related protein/follistatin-like 1 - analysis of their binding with TGF- $\beta$ superfamily proteins. FEBS J 277: 4278-4289, 2010

37. Ouchi N, Asaumi Y, Ohashi K, Higuchi A, Sono-Romanelli S, Oshima Y and Walsh K: DIP2A functions as a FSTL1 receptor. J Biol Chem 285: 7127-7134, 2010.

38. Soffietti R, Rudà R and Trevisan E: Brain metastases: Current management and new developments. Curr Opin Oncol 20: 676-684, 2008. 